\title{
Casamentos Estáveis com Casais Forçados e Casais Proibidos
}

\author{
V.M.F. DIAS ${ }^{1}$, J.L. SZWARCFITER ${ }^{2}$, Programa de Engenharia de Sistemas e \\ Computação, COPPE/UFRJ, Caixa Postal 68511, 21945-970 Rio de Janeiro, RJ, \\ Brasil
}

Resumo. O assunto deste trabalho é o problema dos casamentos estáveis apresentado por Gale \& Shapley [2]. Apresentamos aqui uma caracterização de um casamento estável no qual cada par, de um conjunto de pares dados ("forçados"), forme necessariamente um casal, e nenhum par, de outro dado conjunto de pares ("proibidos"), seja casado. Além disso, apresentamos um algoritmo polinomial que encontra esse casamento. Essa caracterização generaliza um teorema de Gusfield \& Irving [1].

\section{Introdução}

Sejam $H$ e $F$ dois conjuntos disjuntos de cardinalidade $n$, onde cada elemento $h \in H$ é um homem e cada elemento $m \in F$ uma mulher. Um casamento (ou solução) $M$ consiste de $n$ casais monogâmicos $(h, m)$, isto é, uma função bijetora de $H$ em $F$. Suponha que cada homem e cada mulher esteja associado a uma lista de preferência estritamente ordenada contendo todos os elementos do sexo oposto. Um par $(h, m)$ é instável em $M$ se $h$ prefere $m$ que sua parceira em $M$ e $m$ prefere $h$ a seu parceiro em $M$. Nesse sentido, $M$ é um casamento estável se, e somente se, nenhum par em $M$ é instável.

Gale \& Shapley [2] provaram que existe sempre uma solução estável e desenvolveram um algoritmo para encontrá-la em $O\left(n^{2}\right)$. Uma extensão do algoritmo Gale-Shapley encontra todos os casamentos estáveis[4]. Entretanto, Knuth demonstrou em [5] que o número de soluções estáveis para uma instância de tamanho $n$ pode vir a ser exponencial em n. A fim de representar todas as soluções sem necessariamente enumerá-las, Irving \& Leather [3] descreveram uma estrutura de representação compacta de todas as soluções em espaço $O\left(n^{2}\right)$.

Baseando-se nessa representação, o presente trabalho apresenta um algoritmo eficiente para encontrar um casamento estável, caso exista, para solucionar a seguinte extensão do problema. Sejam dados, além de $H, F$ e as listas de preferências, dois conjuntos $Q$ e $P$ de pares $(h, m)$. Um casamento estável $M$ com casais forçados e casais proibidos é tal que todo par de $Q$ é um casal em $M$ e nenhum par

\footnotetext{
$1_{\text {vaniad@ } @ \text { cos.ufrj.br }}$

2 jayme@cos.ufrj.br
} 


$\begin{array}{lllllllll}h_{1} & 3 & 1 & 5 & 7 & 4 & 2 & 8 & 6 \\ h_{2} & 6 & 1 & 3 & 4 & 8 & 7 & 5 & 2 \\ h_{3} & 7 & 4 & 3 & 6 & 5 & 1 & 2 & 8 \\ h_{4} & 5 & 3 & 8 & 2 & 6 & 1 & 4 & 7 \\ h_{5} & 4 & 1 & 2 & 8 & 7 & 3 & 6 & 5 \\ h_{6} & 6 & 2 & 5 & 7 & 8 & 4 & 3 & 1 \\ h_{7} & 7 & 8 & 1 & 6 & 2 & 3 & 4 & 5 \\ h_{8} & 2 & 6 & 7 & 1 & 8 & 3 & 4 & 5\end{array}$

$\begin{array}{lllllllll}m_{1} & 4 & 3 & 8 & 1 & 2 & 5 & 7 & 6 \\ m_{2} & 3 & 7 & 5 & 8 & 6 & 4 & 1 & 2 \\ m_{3} & 7 & 5 & 8 & 3 & 6 & 2 & 1 & 4 \\ m_{4} & 6 & 4 & 2 & 7 & 3 & 1 & 5 & 8 \\ m_{5} & 8 & 7 & 1 & 5 & 6 & 4 & 3 & 2 \\ m_{6} & 5 & 4 & 7 & 6 & 2 & 8 & 3 & 1 \\ m_{7} & 1 & 4 & 5 & 6 & 2 & 8 & 3 & 7 \\ m_{8} & 2 & 5 & 4 & 3 & 7 & 8 & 1 & 6\end{array}$

Figura 1: Instância $I_{8}$ do problema dos casamentos estáveis de tamanho $n=8$

de $P$ forma um casal em $M$. Tal algoritmo foi elaborado a partir de um teorema de caracterização, também formulado no trabalho. Este resultado generaliza um teorema de Gusfield e Irving apresentado em [1].

\section{Representação dos casamentos estáveis}

Em uma instância do problema básico dos casamentos estáveis cada um dos $n$ homens e cada uma das $n$ mulheres estão associados a uma lista de preferência estritamente ordenada contendo todos os elementos do sexo oposto. Aplicando o algoritmo de Gale-Shapley a uma dada instância, obtém-se a solução homem-ótima, isto é, o casamento estável no qual cada um dos homens tem a melhor parceira possível de acordo com sua lista de preferência. Tal solução corresponde a pior para todas as mulheres, também segundo as suas respectivas listas de preferências. Obviamente as regras da aplicação podem ser invertidas obtendo-se assim a solução mulher-ótima.

Sejam $M_{i}$ e $M_{j}$ soluções estáveis. Às operações (i) $M_{i} \vee M_{j}$ e (ii) $M i \wedge M j$ correspondem respectivamente soluções estáveis nas quais (i) todos os homens têm suas piores parceiras entre $M_{i}$ e $M_{j}$, e (ii) todos os homens tem suas parceiras preferidas entre $M_{i}$ e $M_{j}$. Então, $M_{i}$ domina $M_{j}$ de uma perspectiva masculina se, e somente se, todos os homens que possuem parceiras distintas em $M_{i}$ e $M_{j}$ preferem suas parceiras em $M_{i}$ às suas parceiras em $M_{j}$. E ainda, um casamento $M_{k}$ está entre $M_{i}$ e $M_{j}$ se, e somente se, $M_{i}$ domina $M_{k}$ e $M_{k}$ domina $M_{j}$ e, além disso, $M_{k}$ é também uma solução estável (resultados demonstrados em [5, 1]). Na Figura 1, temos um exemplo de uma instância de tamanho $n=8$. Cinco dos casamentos estáveis dessa instância estão representados na Figura 2, entre eles os casamentos $M_{0}$ e $M_{Z}$. Podemos observar que o casamento $M_{0}$ corresponde ao casamento obtido por $M_{1} \wedge M_{2}$ e o resultado da operação $M_{1} \vee M_{2}$ é exatamente o casamento $M_{4}$.

Um reticulado $\mathcal{M}$ representando todos os casamentos estáveis sobre a relação de dominância pode ser construído a partir da solução homem-ótima, denotada por $M_{0}$, onde essa solução é a mais dominante. Por outro lado, a solução mulher-ótima, denotada por $M_{Z}$, em tal representação é dominada por qualquer outra solução em $\mathcal{M}$.

Um casamento $M$ é irredutível quando contém pelo menos um casal $(h, m)$ tal 


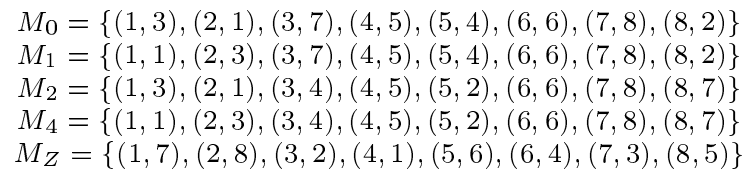

Figura 2: Quatro casamentos estáveis da instância $I_{8}$

que em nenhuma outra solução estável que domine $M, h$ e $m$ formem um casal. Seja $I(\mathcal{M})$ o conjunto de todas as soluções irredutíveis. Então, existe uma ordem parcial $(I(\mathcal{M}), \preceq)$ das soluções de $I(\mathcal{M})$ sobre a relação de dominância herdada de $\mathcal{M}$. Além disso, existe um mapeamento 1-1 entre os subconjuntos fechados de $(I(\mathcal{M}), \preceq)$ e as soluções estáveis em $\mathcal{M}[1]$. A construção de $(I(\mathcal{M}), \preceq)$ pode ser facilmente efetuada em $O\left(n^{5}\right)$. No entanto, omitiremos mais detalhes a respeito desta já que utilizaremos uma outra ordem parcial $(\Pi(\mathcal{M}), \preceq)$ isomorfa a $(I(\mathcal{M}), \preceq)$, removendo-se o elemento minimal desta, que pode ser construída indiretamente em $O\left(n^{2}\right)[1] . I(\mathcal{M})$ e $\Pi(\mathcal{M})$ serão utilizados para denotar os respectivos conjuntos ou ordem parcial de acordo com o contexto. A principal diferença entre as duas ordens é em relação aos seus elementos. Os elementos de $I(\mathcal{M})$ correspondem às soluções irredutíveis, enquanto que os de $\Pi(\mathcal{M})$ são as rotações, abaixo descritas. Em [1] os autores estabelecem uma correspondência entre as duas ordens e mostram ainda como obter $\Pi(\mathcal{M})$ de $I(\mathcal{M})$. Porém, o interesse aqui, por motivo de eficiência, se restringe à construção de $\Pi(\mathcal{M})$ sem o conhecimento prévio de $I(\mathcal{M})$.

$\mathrm{O}$ conceito de rotação é fundamental para o entendimento e construção de $(\Pi(\mathcal{M}), \preceq)$. Seja $M$ um casamento estável. Seja $s_{M}(h)$ a primeira mulher $m$ na lista de $h$ tal que $m$ prefere estritamente $h$ a $p_{M}(m)$, seu parceiro em $M$. E seja $\operatorname{prox}_{M}(h)$ o parceiro em $M$ de $s_{M}(h)$. Então, existe uma cadeia, denominada rotação, de pares ordenados $\pi=\left(h_{0}, m_{0}\right),\left(h_{1}, m_{1}\right), \ldots,\left(h_{r-1}, m_{r-1}\right)$ na solução estável $M$, tal que para cada $i, 0 \leq i \leq r-1, h_{i+1}$ é igual a $\operatorname{prox}_{M}\left(h_{i}\right)$, onde $i+1$ é obtido por $(i$ módulo $r)+1$. Nesse caso, diz-se que a rotação $\pi$ está exposta em $M$. Note que o número máximo de rotações em $\mathcal{M}$ é $n(n-1) / 2$.

Teorema 1 [3] Seja $M$ uma solução estável diferente de $M_{Z}$. Então, existe pelo menos uma rotação exposta em $M$.

A prova deste teorema é construtiva e fornece um método simples de encontrar uma rotação em $M$.

Seja $\pi=\left(h_{0}, m_{0}\right),\left(h_{1}, m_{1}\right), \ldots,\left(h_{r-1}, m_{r-1}\right)$ uma rotação qualquer. Um homem $h$ pertence a $\pi$ se $h=h_{i}$ para algum i, $0 \leq i \leq r-1$. Um par $(h, m)$ pertence a $\pi$ se $h=h_{i}$ e $m=m_{i}$ para algum i, $0 \leq i \leq r-1$. Seja $M$ uma solução estável e $\pi$ exposta em $M$, então $M / \pi$ é definida como sendo a solução na qual cada homem não pertinente a $\pi$ continua casado com sua parceira em $M$, e cada homem que pertence a $\pi$ tem como parceira $m_{i+1}=s_{M}\left(h_{i}\right)$. Tal operação é chamada de eliminação.

Teorema 2 [3] Toda solução estável $M$ pode ser gerada por uma seqüência de eliminações de rotações, a partir de $M_{0}$, e todas as seqüências entre $M_{0}$ e $M$ contêm exatamente as mesmas rotações. 
Um par $(h, m)$ é um casal estável se $h$ e $m$ são parceiros em algum casamento estável $M$. Agora, se $h$ e $m$ são casados em todo casamento estável $M$ de $\mathcal{M}$, então $(h, m)$ é um casal fixo.

Teorema 3 [1] (i) Um par $(h, m)$ é um casal estável se, e somente se, é um casal em $M_{Z}$ ou pertence a alguma rotação. Equivalentemente, $(h, m)$ é estável se, e somente se, é par em $M_{0}$ ou para alguma rotação $\pi=\left(h_{0}, m_{0}\right),\left(h_{1}, m_{1}\right), \ldots,\left(h_{r-1}, m_{r-1}\right)$ e para algum $i, h=h_{i}$ e $m=m_{i+1}$.

(ii) Um casal é fixo se, e somente se, é um casal em $M_{0}$ e em $M_{Z}$. Equivalentemente, se é um casal em $M_{0}$ e não pertence a nenhuma rotação.

Uma consequência direta dos teoremas anteriores é que as soluções estáveis em uma cadeia maximal em $\mathcal{M}$, isto é, uma seqüência de casamentos estáveis consecutivos em $\mathcal{M}$ com início em $M_{0}$ e término em $M_{Z}$, contém todos os pares estáveis de $\mathcal{M}$. Além disso, uma rotação $\pi^{\prime}$ precede uma rotação $\pi$ em $\Pi(\mathcal{M})$ se, e somente se, $\pi^{\prime}$ aparece antes de $\pi$ em toda cadeia maximal em $\mathcal{M}$.

Seja $\Pi(\mathcal{M})$ o conjunto de todas as rotações expostas em $\mathcal{M}$. Então, existe uma ordem parcial $(\Pi(\mathcal{M}), \preceq)$ dos elementos de $\Pi(\mathcal{M})$ sobre a relação de precedência herdada de $\mathcal{M}$

Teorema 4 [3] Existe um mapeamento 1-1 entre os subconjuntos fechados de $(\Pi(\mathcal{M}), \preceq)$ e as soluções estáveis em $\mathcal{M}$.

A construção de $\Pi(\mathcal{M})$ é descrita aqui em linhas gerais.

(i) Encontrar todas as rotações realizadas em $O\left(n^{2}\right)$ : a partir de $M=M_{0}$, repete-se sucessivamente, encontrar $\pi$ exposta em $M$, eliminar $\pi$ obtendo $M=$ $M / \pi$, até $M=M_{Z}$. ( Note que a eliminação de $\pi$ é proporcional ao número de homens em $\pi$ que é $O(n))$.

(ii) Construir um grafo $G(\mathcal{M})$, onde $G(\mathcal{M})$ é um subconjunto de pares de rotações na ordem parcial $\Pi(\mathcal{M})$ tal que o fechamento transitivo de $G(\mathcal{M})$ é a ordem parcial $\Pi(\mathcal{M})$. O grafo $G(\mathcal{M})$ é definido por duas regras de precedência relativas aos pares $(h, m)$ em cada rotação, o que pode ser visto em maiores detalhes em [1], bem como a equivalência entre o fechamento transitivo de $G(\mathcal{M})$ e a ordem parcial $\Pi(\mathcal{M})$. A construção de $G(\mathcal{M})$, a partir das rotações, também é realizada em $O\left(n^{2}\right)$.

Tanto (i) como (ii) são totalmente determinados pelas listas de preferências. Várias derivações do problema dos casamentos estáveis podem fazer uso do digrafo $G(\mathcal{M})$ e da ordem parcial $\Pi(\mathcal{M})$, a fim de obter algoritmos mais eficientes em relação às suas complexidades. Vamos examinar na seção seguinte o problema de determinar se um dado conjunto de pares é um conjunto estável[1]. Na seção 4 é apresentada uma extensão desse problema e, na seção 5 , o algoritmo correspondente.

A Figura 4 mostra o reticulado $\mathcal{M}$. À direita de cada ligação entre um par de casamentos $\left(M, M^{\prime}\right)$ está indicada uma rotação $\pi$ exposta em $M$. Ou seja, o casamento $M^{\prime}$ pode ser obtido a partir de $M$ pela eliminação de $\pi$. Além disso, todas as rotações da instância $I_{8}$ estão representadas na Figura 3. Pode-se verificar que, a toda cadeia, entre dois casamentos quaisquer $M$ e $M^{\prime}$ corresponde o mesmo 


$$
\begin{aligned}
& \pi_{1}=\{(1,3),(2,1)\} \\
& \pi_{2}=\{(3,7),(5,4),(8,2)\} \\
& \pi_{3}=\{(4,5),(7,8),(6,6)\} \\
& \pi_{4}=\{(1,1),(6,5),(8,7)\} \\
& \pi_{5}=\{(2,3),(3,4)\} \\
& \pi_{6}=\{(4,8),(7,6),(5,2)\} \\
& \pi_{7}=\{(3,3),(8,1)\} \\
& \pi_{8}=\{(2,4),(5,8),(6,7)\} \\
& \pi_{9}=\{(1,5),(5,7),(8,3)\} \\
& \pi_{10}=\{(3,1),(7,2),(5,3),(4,6)\}
\end{aligned}
$$

Figura 3: Rotações em $\mathcal{M}$ da instância $I_{8}$

conjunto de rotações. Em particular, toda cadeia maximal de $I_{8}$ compreende o conjunto $\left\{\pi_{1}, \pi_{2}, \pi_{5}, \pi_{3}, \pi_{4}, \pi_{7}, \pi_{6}, \pi_{8}, \pi_{9}, \pi_{10}\right\}$.

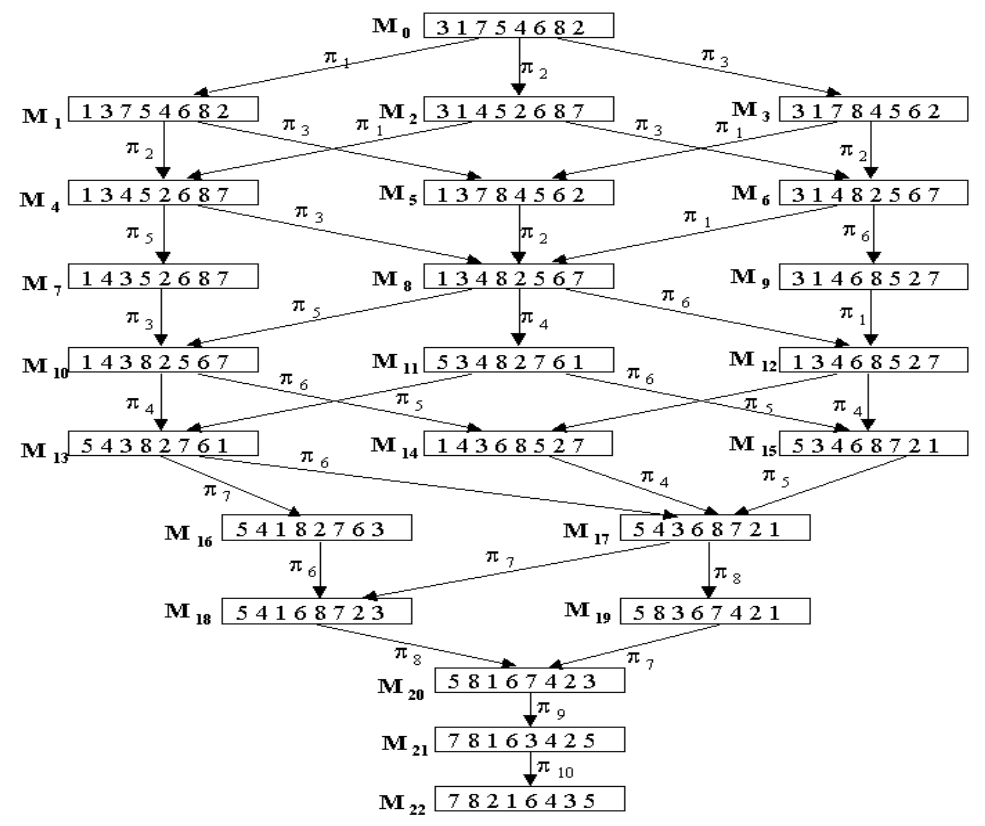

Figura 4: Casamentos estáveis da instância $I_{8}$ com rotações 


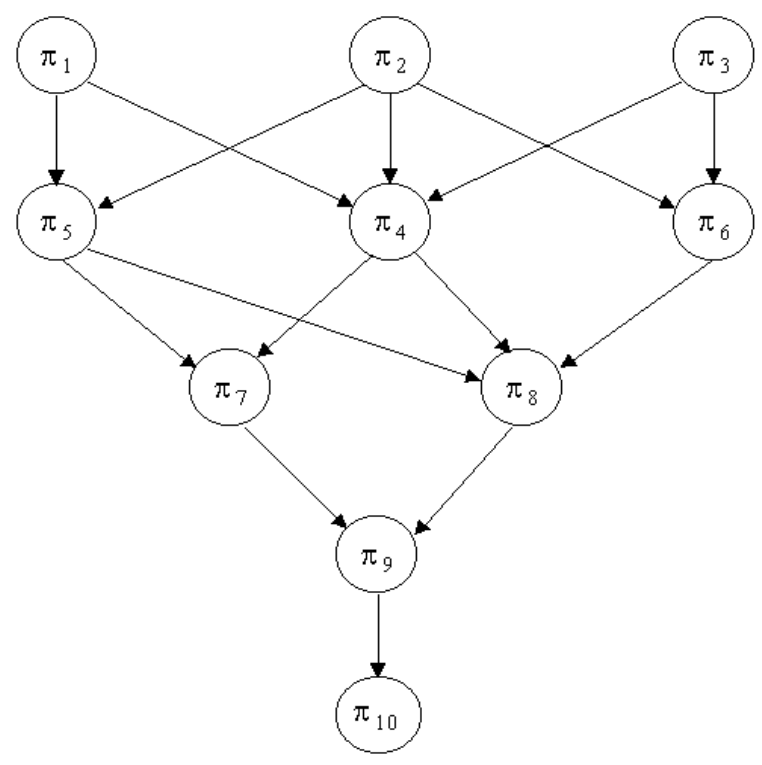

Figura 5: Conjunto parcialmente ordenado de rotações $(\Pi(\mathcal{M}), \preceq)$ da instância $I_{8}$

O conjunto parcialmente ordenado de rotações da instância $I_{8}$ é apresentado na Figura 5. Como visto, cada casamento $M$ de $I_{8}$ corresponde a um subconjunto fechado de $(\Pi(\mathcal{M}), \preceq)$. Por exemplo, o casamento $M_{13}$ é obtido pela eliminação das rotações no conjunto $\left\{\pi_{1}, \pi_{2}, \pi_{3}, \pi_{4}, \pi_{5}\right\}$.

\section{Casais Forçados}

Um conjunto $Q$ de pares $(h, m)$ é um conjunto de casais forçados se existe algum casamento estável $M$ tal que todo par em $Q$ é um casal em $M$. Para cada par $(h, m)$ em $Q$, não casado em $M_{0}$, define-se $\gamma(h, m)$ como sendo a única rotação que move $h$ para $m$, isto é, tal que $h=h_{i}$ e $m=m_{i+1}$, para algum i, na rotação $\pi=\gamma(h, m)$ e, para cada par de $Q$ não casado em $M_{Z}$, define-se $\theta(h, m)$ como a única rotação que move $h$ a partir de $m$, ou ainda, cujo par $(h, m)$ pertence à $\pi=\theta(h, m)$.

Teorema 5 [1] Um conjunto $Q$ de pares é um conjunto estável (casais forçados) se, e somente se, (i) cada par é estável e (ii) não existem dois pares $(h, m)$ e $\left(h^{\prime}, m^{\prime}\right)$ em $Q$ tais que $\theta(h, m) \prec \gamma\left(h^{\prime}, m^{\prime}\right)$ em $\Pi(\mathcal{M})$.

Como visto anteriormente, todas as rotações podem ser encontradas em $O\left(n^{2}\right)$. Conhecendo-se as rotações e com base no teorema de caracterização de pares estáveis, temos que todos os pares estáveis de uma dada instânica podem ser encontrados em $O\left(n^{2}\right)$. A determinação das rotações $\theta(h, m)$ e $\gamma(h, m)$ para todos os pares estáveis também é facilmente realizada em $O\left(n^{2}\right)$. No entanto, o problema em questão é um dos poucos no qual $\Pi(\mathcal{M})$ não pode ser substituído por $G(\mathcal{M})$. Logo, exige a 


$\begin{array}{ccccccccc}(h, m) & \gamma & \theta & (h, m) & \gamma & \theta & (h, m) & \gamma & \theta \\ (1,3) & \emptyset & \pi_{1} & (3,4) & \pi_{2} & \pi_{5} & (3,1) & \pi_{7} & \pi_{10} \\ (2,1) & \emptyset & \pi_{1} & (4,8) & \pi_{3} & \pi_{6} & (7,2) & \pi_{6} & \pi_{10} \\ (3,7) & \emptyset & \pi_{2} & (7,6) & \pi_{3} & \pi_{6} & (5,3) & \pi_{9} & \pi_{10} \\ (5,4) & \emptyset & \pi_{2} & (5,2) & \pi_{2} & \pi_{6} & (4,6) & \pi_{6} & \pi_{10} \\ (8,2) & \emptyset & \pi_{2} & (3,3) & \pi_{5} & \pi_{7} & (1,7) & \pi_{9} & \emptyset \\ (4,5) & \emptyset & \pi_{3} & (8,1) & \pi_{4} & \pi_{7} & (2,8) & \pi_{8} & \emptyset \\ (7,8) & \emptyset & \pi_{3} & (2,4) & \pi_{5} & \pi_{8} & (3,2) & \pi_{10} & \emptyset \\ (6,6) & \emptyset & \pi_{3} & (5,8) & \pi_{6} & \pi_{8} & (4,1) & \pi_{10} & \emptyset \\ (1,1) & \pi_{1} & \pi_{4} & (6,7) & \pi_{4} & \pi_{8} & (5,6) & \pi_{10} & \emptyset \\ (6,5) & \pi_{3} & \pi_{4} & (1,5) & \pi_{4} & \pi_{9} & (6,4) & \pi_{8} & \emptyset \\ (8,7) & \pi_{2} & \pi_{3} & (5,7) & \pi_{8} & \pi_{9} & (7,3) & \pi_{10} & \emptyset \\ (2,3) & \pi_{1} & \pi_{5} & (8,3) & \pi_{7} & \pi_{9} & (8,5) & \pi_{9} & \emptyset\end{array}$

Figura 6: Rotações $\theta$ e $\gamma$ para os pares estáveis de $I_{8}$

construção explícita de $\Pi(\mathcal{M})$, realizada em $O\left(n^{4}\right)$ a partir de $G(\mathcal{M})$. Seja $|Q|=k$, a condição (ii) pode ser verificada em $O\left(k^{2}\right)$.

Teorema 6 [1] Após o pré-processamento, realizado em $O\left(n^{4}\right)$, a estabilidade de um conjunto $Q$ com $k$ pares pode ser determinada em tempo $O\left(k^{2}\right)$.

Na Figura 6, cada par estável $(h, m)$ de $I_{8}$ tem listadas, à sua direita, duas rotações. A primeira delas é $\gamma(h, m)$, cuja eliminação une tal par, e a outra é $\theta(h, m)$, cuja eliminação o separa.

Considere o conjunto $Q=\{(1,5),(2,8),(6,6)\}$ para a instância $I_{8}$. O par $(6,6)$ é casado em $M_{0}$ e é separado pela eliminação da rotação $\pi_{3}$, ou seja, $\pi_{3}=\theta(6,6)$. Mas, $\pi_{3} \prec \pi_{4}$ e $\pi_{4}=\gamma(1,5)$. Claramente, antes da eliminação de $\pi_{4}$ o par $(1,5)$ não forma um casal para nenhum $M$. Logo, $Q$ não é um conjunto estável em $I_{8}$. Agora, seja $Q^{\prime}=\{(1,5),(2,8),(6,4)\}$. A única rotação que separa um desses pares é $\pi_{9}=$ $\theta(1,5)$. A rotação $\pi_{8}$ é a última que une um desses pares $\left(\pi_{8}=\gamma(2,8)=\gamma(6,4)\right)$. Como $\pi_{9} \npreceq \pi_{8}$, concluímos que existe um casamento estável com casais forçados $Q^{\prime}$. O casamento dominante que satisfaz $Q^{\prime}$ é $M_{19}=\{(1,5),(2,8),(3,3),(4,6),(5,7)$, $(6,4),(7,2),(8,1)\}$, que corresponde ao subconjunto fechado $\left\{\pi_{1}, \pi_{2}, \pi_{3}, \pi_{4}, \pi_{5}\right.$, $\left.\pi_{6}, \pi_{8}\right\}$.

\section{Casais Forçados e Casais Proibidos}

Nessa seção apresentaremos uma extensão do problema anterior.

Um par $(h, m)$ é um casal proibido em $\mathcal{M}$ se existe um casamento estável $M$ tal que $(h, m)$ não seja um casal em $M$. Um conjunto de pares $P$ é um conjunto de 
casais proibidos se existe um casamento estável $M$ no qual nenhum par de $P$ forma um casal em $M$.

Lema 7 Um par $(h, m)$ é um casal proibido se, e somente se, $(h, m)$ não é fixo.

A prova segue diretamente da definição e do teorema de caracterização de pares estáveis.

Seja $S$ um subconjunto fechado de $\Pi(\mathcal{M})$. Então $\pi$ é uma rotação maximal em $S$ se $\pi$ não é sucedida por nenhuma rotação em $S$.

Teorema 8 Um conjunto de pares $P$ é um conjunto de casais proibidos se, e somente se, $(i)$ nenhum dos pares de $P$ é fixo e (ii) existe um subconjunto fechado $S$ de $\Pi(\mathcal{M})$ tal que para todo par $(h, m)$ de $P$ em $M_{0}$ a rotação $\theta(h, m)$ pertence a $S$ e, para todo par $(h, m)$ em $P$ não contido em $M_{0}$, se $\gamma(h, m)$ pertence a $S$, então deve existir $\theta(h, m)$ em $S$.

Demonstração: $(\Rightarrow)$ Claramente, sendo $P$ um conjunto de casais proibidos, nenhum par de $P$ pode ser fixo. Seja $M$ um casamento com casais proibidos $P$. A $M$ corresponde um único subconjunto fechado $S$ de $\Pi(\mathcal{M})$. Seja $(h, m)$ um par de $P$ que forma um casal em $M_{0}$, ou ainda, tal que $\gamma(h, m) \in S$. Como $h$ e $m$ não são casados em $M$, temos que $\theta(h, m) \in S$.

$(\Leftarrow)$ Seja $S$ um subconjunto fechado de $\Pi(\mathcal{M})$ tal que para todo par $(h, m) \in P$ se $(h, m)$ é um casal em $M_{0}$ ou $\gamma(h, m) \in S$ então $\theta(h, m) \in S$. Seja $M$ o casamento correspondente a $S$. Suponha que, para algum par $(h, m)$ de $P,(h, m)$ forma um casal em $M$. Nesse caso, $\gamma(h, m) \in S$ ou $(h, m) \in M_{0}$, e, $\theta(h, m) \in S$, assim temos uma contradição.

Seja $P=\{(1,1),(1,5),(2,1),(2,8)\}$ um candidato a conjunto de casais proibidos em $I_{8}$. Inicialmente, verificamos que nenhum par de $P$ é fixo. O par $(2,1)$ é casado em $M_{0}$ e é separado pela eliminação da rotação $\pi_{1}$, ou seja, $\pi_{1}=\theta(2,1)$. Mas, $\pi_{1}=\gamma(1,1)$. Esse casal é separado durante a eliminação da rotação $\pi_{4}=\theta(1,1)$, que por outro lado une o par $(1,5)$, isto é, $\pi_{4}=\gamma(1,5)$. Agora, $\pi_{9}=\theta(1,5)$ e $\pi_{8} \prec \pi_{9}$. Como $\pi_{8}=\gamma(2,8)$ e não existe rotação cuja eliminação separe tal par, concluímos que não existe subconjunto fechado de $\Pi(\mathcal{M})$ que satisfaça à condição (ii) do Teorema 3.5. Logo, $P$ não é um conjunto de casais proibidos em $I_{8}$. Agora, seja $P^{\prime}=\{(1,1),(1,5),(2,1),(3,3)\}$. Para o par $(3,3)$, tem-se que $\pi_{5}=\gamma(3,3)$ e $\pi_{7}=\theta(3,3)$. Assim, o subconjunto $S=\left\{\pi_{1}, \pi_{2}, \pi_{3}, \pi_{4}, \pi_{5}, \pi_{6}, \pi_{7}, \pi_{8} \pi_{9}\right\}$ satisfaz (ii). Logo, $P^{\prime}$ é um conjunto de casais proibidos em $I_{8}$. O casamento $M_{21}=$ $\{(1,7),(2,8),(3,1),(4,6),(5,3),(6,4),(7,2),(8,5)$ que corresponde a $S$ em $\mathcal{M}$ é um casamento no qual nenhum dos pares de $P^{\prime}$ é casado.

Combinando os Teoremas 5 e 8, obtemos a seguinte caracterização de casamento estável com casais forçados $Q$ e casais proibidos $P$.

Teorema 9 Sejam $P$ um conjunto de casais proibidos e $Q$ um conjunto de casais forçados em $\mathcal{M}$. Então, existe uma solução estável $M$ em $\mathcal{M}$ que satisfaz $Q$ e $P$ se, e somente se, existe um subconjunto fechado $S$ de $\Pi(\mathcal{M})$ que $(i)$ satisfaz $Q$ e 
(ii) para quaisquer dois pares $(h, m) \in P$ e $\left(h^{\prime}, m^{\prime}\right) \in Q$, se não existe $\theta(h, m) \in S$ ou $\theta\left(h^{\prime}, m^{\prime}\right) \preceq \theta(h, m)$ em $\Pi(\mathcal{M})$ então $\gamma(h, m) \notin S$ nem é um casal em $M_{0}$.

Demonstração: $(\Rightarrow)$ Seja $M$ um casamento estável com casais proibidos $P$ e casais forçados $Q$. Seja $S$ o subconjunto fechado de $\Pi(\mathcal{M})$ correspondente à $M$. Obviamente, nenhum par em $P$ é fixo e todo par de $Q$ é estável.

(i) Seja $\left(h^{\prime}, m^{\prime}\right)$ um par de $Q$ não casado em $M_{0}$. Então, $\gamma\left(h^{\prime}, m^{\prime}\right)$ existe e pertence à $S$. Agora, se para algum par $(h, m), \theta(h, m) \preceq \gamma\left(h^{\prime}, m^{\prime}\right)$ em $\Pi(\mathcal{M})$ então $\theta(h, m) \in S$ e o par $(h, m)$ não é um casal em $M$. Logo, para quaisquer dois pares de $Q \theta \npreceq \gamma$.

(ii) Seja $(\mathrm{h}, \mathrm{m})$ um par de $P$ tal que $\theta\left(h^{\prime}, m^{\prime}\right) \preceq \theta(h, m)$ em $\Pi(\mathcal{M})$. Como $h^{\prime}$ e $m^{\prime}$ são casados em $M, \theta\left(h^{\prime}, m^{\prime}\right) \notin S$, $\log \theta \theta(h, m) \notin S$. Mas, como $h$ e $m$ não são casados em $M$, concluímos que $\gamma(h, m) \notin S$, bem como $h$ e $m$ não formam um casal em $M_{0}$. Por outro lado, se $\gamma(h, m) \in S$ então $\theta(h, m)$ existe e deve estar em $S$. Como todo par $\left(h^{\prime}, m^{\prime}\right)$ de $Q$ é um casal em $M$ temos que para nenhum $\left(h^{\prime}, m^{\prime}\right)$, $\theta\left(h^{\prime}, m^{\prime}\right) \preceq \theta(h, m)$ em $\Pi(\mathcal{M})$.

$(\Leftarrow)$ Seja $S$ o menor subconjunto fechado de $\Pi(\mathcal{M})$ que satisfaz às condições (i) e (ii). Então, as rotações maximais em $S$ são do tipo $\gamma$ em relação aos pares de $Q$, ou do tipo $\theta$ para os pares de $P$. Seja $M$ o casamento estável correspondente a $S$. Todo par $\left(h^{\prime}, m^{\prime}\right)$ de Q não casado em $M_{0}$ é casado em $M$, pois se $\gamma\left(h^{\prime}, m^{\prime}\right)$ é maximal então $\theta\left(h^{\prime}, m^{\prime}\right) \notin S$. Caso contrário, seja $\theta(h, m)$ uma rotação maximal em $S$ tal que $\gamma\left(h^{\prime}, m^{\prime}\right) \prec \theta(h, m)$. Então, por (ii), $\neg \exists\left(h^{\circ}, m^{\circ}\right)$ de $Q$ tal que $\theta\left(h^{\circ}, m^{\circ}\right) \preceq \theta(h, m)$. Logo, $\theta\left(h^{\prime}, m^{\prime}\right) \notin S$ (analogamente para $\left.\left(h^{\prime}, m^{\prime}\right) \in M_{0}\right)$.

Falta mostrar que nenhum par $(h, m) \in P$ é um casal em $M$. Pela contrapositiva de (ii), se $\gamma(h, m) \in S$ ou $(h, m) \in M_{0}$ então $\theta(h, m) \in S$. Logo, $h$ e $m$ não são casados em $M$.

\section{Algoritmo}

Baseado no teorema anteriormente apresentado, descrevemos, na Figura 7, o algoritmo FP para encontrar um casamento estável com casais forçados $Q$ e casais proibidos $P$. O pré-processamento requerido aqui é o mesmo especificado na seção 3 , acrescendo-se a verificação de que $Q$ é de fato um conjunto de casais forçados em $\mathcal{M}$, realizado em $O\left(k^{2}\right)$. O tempo total do pré-processamento é portanto $O\left(n^{4}\right)$. 
Algoritmo FP

Entrada:

Conjuntos $P$ e $Q$;

Solução $M_{0}$;

Ordem parcial $\Pi(\mathcal{M})$;

\section{Início}

Remover os pares não estáveis de $P$;

Para cada $\pi \in S$ faça $S[\pi] \leftarrow$ falso;

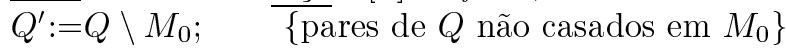

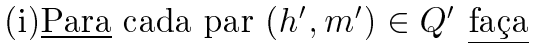

Seja $\pi$ tal que $\pi$ é $\gamma\left(h^{\prime}, \overline{\left.m^{\prime}\right) \text {; }}\right.$

Incluir $\pi$ e seus precedentes em $S$;

(a) $P_{0}:=P \cap M_{0}$;

(b) $P^{\prime}:=\left\{(h, m) \in P_{0}: \theta(h, m) \notin S\right\}$;

(c) $P^{\prime}:=P^{\prime} \cup\{(h, m) \in P: \gamma(h, m) \in S$ e $\theta(h, m) \notin S\}$;

(ii)Enquanto $P^{\prime}$ não for vazio faça

Para cada par $(h, m)$ de $\overline{P^{\prime}}$ faça

Seja $\pi$ tal que $\pi$ é $\theta(h, \overline{m) ;}$

Se $\pi \neq n$ nulo então

(ii.1)Incluir $\pi$ e seus precedentes em $S$ caso contrário

"Não há solução que satisfaça $Q$ e $P$ " $\rightarrow$ fim

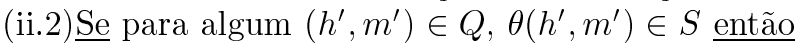

"Não há solução que satisfaça $Q$ e $P$ " $\rightarrow$ fim

caso contrário

$P \leftarrow P \backslash P^{\prime}$

fim_enqto;

(ii.3) $P^{\prime}:=\{(h, m) \in P: \gamma(h, m) \in S$ e $\theta(h, m) \notin S\}$;

\section{Fim}

Figura 7: Algoritmo que encontra o casamento minimal que satisfaz $\mathrm{P}$ e Q 
O algoritmo tem como entrada dois conjuntos de pares $P$ e $Q$, respectivamente conjuntos de casais proibidos e forçados, representados por matrizes, o casamento estável $M_{0}$ e a ordem parcial $\Pi(\mathcal{M})$. A variável $P_{0}$ consiste dos pares de $P$ casados em $M_{0}$. A saída do algoritmo é um subconjunto fechado $S$ de $\Pi(\mathcal{M})$ que satisfaz $Q$ e $P$. $S$ é inicializado como vazio, em (i) passa a corresponder ao subconjunto mínimo que satisfaz $Q$ e é atualizado durante a execução de (ii), de acordo com a variável $P^{\prime}$ (em cada iteração) que consiste dos pares $(h, m)$ de $P$ casados em $M_{0}$ ou ainda cuja rotação $\gamma(h, m) \in S$ e tal que a rotação $\theta(h, m) \notin S$. A fim de melhorar a complexidade, $S$ deve ser uma lista de valores lógicos, onde a cada índice de $S$ corresponde uma rotação.

Sejam $r$ o número de rotações em $\Pi(\mathcal{M}), k$ e $l$, as cardinalidades de $Q$ e $P$, respectivamente, e $n$ o tamanho da instância do problema. Encontrar o subconjunto minimal que satisfaz $Q$, é executado por (i) em tempo $O(k . r)$. Os pares de $P_{0}$ (a) podem ser encontrados em tempo $O(n)$. Tanto (b) como (c) são lineares no tamanho de $P$. Logo, realizados em tempo $O(l)$. Em (ii), no pior caso, se um par de $P$ é removido a cada iteração, para o último par removido é verificado $l$ vezes se $\gamma(h, m) \in S$ e $\theta(h, m) \notin S$ (ii.3), um tempo máximo de $O\left(l^{2}\right)$. Para cada par de $P$ são incluídas em $S$ as rotações $\theta(h, m)$ e suas precedentes (ii.1), consumindo um tempo total de $O(r . l)$. Ainda, deve ser verificado se tal inclusão viola a satisfatibilidade de $Q$, isto é, se alguma rotação incluída em $S$ é do tipo $\theta$ para algum par de $Q$ (ii.2), realizado em tempo $O(k)$. Portanto, (ii) despende um tempo total de $O(l . k)$. Finalmente, obter a solução $M$ correspondente a $S$ pode ser realizado em $O\left(n^{2}\right)$. Como visto anteriormente, o número de rotações $r$ é $O\left(n^{2}\right)$. Pela definição do problema, o valor máximo de $k$ é $n$, e $l$ não pode ultrapassar $n^{2}$. Logo, a complexidade total do algoritmo FP é $O\left(n^{4}\right)$.

\section{Referências}

[1] D. Gusfield and R.W. Irving, "Stable Marriage Problem - Structure and Algorithms", The MIT Press, 1989.

[2] D. Gale and L.S. Shapley, College admissions and the stability of marriage, American Mathematical Monthly, 69 (1962), 9-15.

[3] R.W. Irving and P. Leather, The complexity of counting stable marriages, SIAM J. Comput., 15 (1986), 655-667.

[4] D. McVitie and L.B. Wilson, The stable marriage problem, Commun. of the A.C.M., 14 (1971), 486-490.

[5] D.E. Knuth. "Marriages Stables", Les Presses de l'Université de Montreal, 1976.

[6] R.W. Irving, P. Leather and D. Gusfield, An efficient algorithm for the 'optimal' stable marriage, Journal of the A.C.M., 34 (1987), 532-543. 
